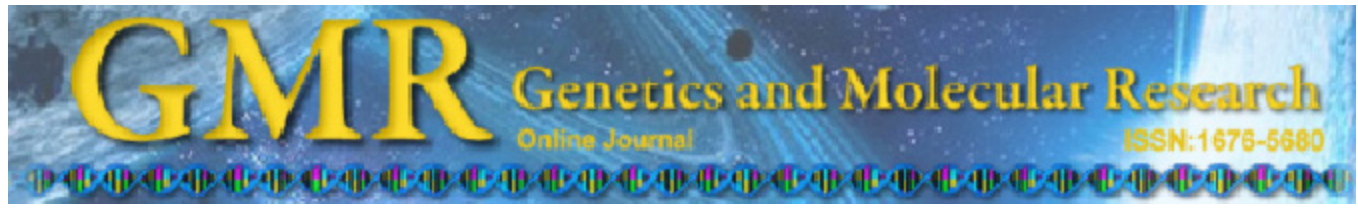

\title{
Analysis of thyroid peroxidase antibody in early pregnancy
}

\author{
L. Lin, X.L. Zhang and Y. Long \\ Department of Gynecology \& Obstetrics, \\ Beijing Friendship Hospital Affiliated to Capital Medical University, \\ Beijing, China \\ Corresponding author: Y. Long \\ E-mail: yanlongcn@yeah.net \\ Genet. Mol. Res. 13 (3): 5107-5114 (2014) \\ Received May 21, 2013 \\ Accepted October 4, 2013 \\ Published July 7, 2014 \\ DOI http://dx.doi.org/10.4238/2014.July.7.3
}

ABSTRACT. This study aimed to investigate the thyroid peroxidase
antibody (TPOAb) positive rate and how TPOAb affects thyroid
function between 7 and 12 weeks of gestation. Six hundred and
eleven cases of healthy primipara were enrolled in this study between
September 2010 and June 2011 , and all of them were given a regular
prenatal check in Beijing Friendship Hospital. By detecting the levels
of thyroid stimulating hormone (TSH), free thyroxine (FT4), and
TPOAb between 7 to 12 weeks gestation, we developed an early thyroid
function normal reference interval during pregnancy. Additionally, we
analyzed the TPOAb-positivity cutoff value, the positive rate, and the
affect of TSH and FT4. We found that the TPOAb median values and
changes in the range were 38.9 (6.4-1300+) mU/L. By establishing an
$7-12$ week gestational-specific reference standard for pregnancy, we set
a positive cutoff value of 206.77 mU/L according to the 90 th percentile
TPOAb. The TPOAb positive rate was $10.8 \%$ (66/611). Regression
analysis showed that, during gestation between 7 and 12 weeks, the
titers of TPOAb and TSH correlate positively and negatively with FT4,
$\beta=1.406$ and 0.820 , respectively [ $95 \%$ CI (1.349-1.413, $0.805-0.835$ ),
$\mathrm{P}=0.000$ ]. Pregnant TPOAb positive women are more likely to suffer 
from markedly increased TSH levels, with increased risk being 3-4-fold greater than in a normal pregnancy.

Key words: Early stage of pregnancy; Thyroid peroxidose antibody; Gestational age-specific thyroid function reference interval

\section{INTRODUCTION}

Thyroid peroxidase antibody (TPOAb) is a serological marker of autoimmune thyroid disease (AITD), which more commonly affects pregnant women. Hence, TPOAb is associated with adverse pregnancy outcomes (Lepoutre et al., 2012). Previous studies show and report that the TPOAb positive rate of early pregnancy is quite different and ranges from 5 to $17 \%$ (Haddow et al., 2010; Männistö et al., 2011). This is mainly due to the lack of pregnancy TPOAb-positive criteria. In addition, this variation in the TPOAb positive rate also relates to differences in race, age, and laboratory testing methods. There were 611 healthy primipara included in this study, and all of them carried out prenatal checks at the Beijing Friendship Hospital obstetric outpatient center from September 2010 to June 2011.

For the pregnant women mentioned above, thyroid function and TPOAb were detected at 7 to 12 weeks. Accordingly, the specific thyroid function reference standard and a TPOAb positive cutoff value were established for the primipare who received a prenatal check in Beijing Friendship Hospital from 7 to 12 gestational weeks. Moreover, the effects of TPOAb on thyroid stimulating hormone (TSH) and free thyroxin (FT4) levels were analyzed and explored. Hopefully, this study can provide a reference for clinicians.

\section{MATERIAL AND METHODS}

\section{Object of study}

There were 611 healthy primipara included in this study, and all of them received a prenatal check at the Beijing Friendship Hospital obstetric outpatient center from September 2010 to June 2011 (they denied having any chronic diseases, history of thyroid disease, family history of thyroid disease, or adverse birth history). They ranged in age from 21 to 41 years old $(29 \pm 3.08)$. All had a single pregnancy. Thyroid function (TSH, FT4) and TPOAb were detected at 7 to 12 weeks of gestation. Furthermore, the median urinary iodine level was $150.31 \mathrm{~g} / \mathrm{L}$.

\section{General data collection}

The study subjects participated in a questionnaire survey project, which included questions regarding whether they had earlier suffered from chronic diseases or not, reproductive history, personal and family history of thyroid disease, iodine deficiency, life history, diet habits, and other factors.

\section{Laboratory tests}

We obtained $3 \mathrm{~mL}$ blood from fasting pregnant women and then separated the serum. We used the chemiluminescence immunoassay to test serum TSH, FT4, and TPOAb levels. TSH and 
FT4 measurements were carried out using Beckman (BECKMAN COULTER Unicel DXI 800, USA) instruments and kits. The determination of TPOAb levels was carried out using Centaur instruments and kit (ADVIA Centaur, Germany). After testing, our data showed that both inter- and intra-assay variation was $<6 \%$. The maximum detection range of TPOAb was $<1300 \mathrm{mU} / \mathrm{L}$.

\section{TPOAb positive cutoff value and the normal reference range}

Following manufacturer protocols, reference ranges of TPOAb were obtained to be between 0 and $60 \mathrm{mIU} / \mathrm{L}$, and the positive cutoff value was $60 \mathrm{mIU} / \mathrm{L}$. In this study, gestational crowd-specific reference standards were used. Methods were as follows: 1) Initially, we ensured that the study population had a normal TSH reference interval. According to the recommendation of the NACB (National Academy of Clinical Biochemistry), we set the range of TSH as the $95 \%$ confidence intervals of a "standard population". The conditions of a "standard population" in pregnancy excluded a history of thyroid disease and TPOAb levels greater than the reference value provided by the manufacturer $(60 \mathrm{mIU} / \mathrm{L})$. 2) The study population complies with TSH in the 95th percentile of the normal range of pregnant women. We used the 90th percentile of the normal range to calculate the TPOAb positive cutoff value and normal reference range.

\section{Statistical methods}

All findings and laboratory data were entered into a database, and we used the SPSS software for statistical analysis. ANOVA (continuous variables), $\chi^{2}$ or Fisher's exact test (categorical variables), and Spearman rank correlation were used to analyze the data.

The values between groups were analyzed by independent sample T test. A P value less than 0.05 was considered to be statistically significant.

\section{RESULTS}

\section{Six hundred and eleven cases of healthy primipara thyroid function and TPOAb check up results (7-12 weeks gestation)}

Table 1 shows the median value, range of change, and percentiles of TSH, FT4, and TPOAb, respectively.

\begin{tabular}{|c|c|c|c|c|c|c|c|}
\hline Items & Median & Min. & Max. & 2.5 percentile & 5 percentile & 95 percentile & 97.5 percentile \\
\hline TSH (mU/L) & 1.12 & 0.01 & 7.38 & 0.03 & 0.10 & 3.38 & 4.15 \\
\hline FT4 (pM) & 10.68 & 6.69 & 89.58 & 7.21 & 8.11 & 15.06 & 18.15 \\
\hline TPOAb (mU/L) & 38.9 & 6.40 & 1300 & 12.89 & 15.62 & 630.0 & 1300 \\
\hline
\end{tabular}

$\mathrm{TSH}=$ thyroid stimulating hormone; FT4 = free thyroxine; TPOAb $=$ thyroid peroxidase antibody.

\section{Reference normal range of thyroid function according to 471 cases of a "standard population" that are pregnant from 7 to 12 weeks}

The study included 611 women in early pregnancy, of which 471 cases were used 
as a "standard population". Two conditions were excluded: a previous history of thyroid disease and TPOAb $>60 \mathrm{mU} / \mathrm{L}$. Table 2 shows the median value, range of change, and percentiles of TSH, FT4, and TPOAb, respectively, among 471 "standard population" patients. Based on data shown in Table 2, we know that the normal reference range for TSH was between 0.03 and $3.67 \mathrm{mU} / \mathrm{L}$, and the reference value range of FT4 was between 7.21 and $18.66 \mathrm{pmol} / \mathrm{L}$. All data were collected between 7 and 12 weeks gestation.

Table 2. TSH, FT4 median value and percentile of 471 cases standard crowd in 7 to 12 weeks gestation.
\begin{tabular}{|cccccccc}
\hline Items & Median & Min. & Max. & 2.5 percentile & 5 percentile & 95 percentile & 97.5 percentile \\
\hline TSH $(\mathrm{mU} / \mathrm{L})$ & 1.12 & 0.01 & 7.01 & 0.03 & 0.03 & 3.03 & 3.67 \\
FT4 $(\mathrm{pmol} / \mathrm{L})$ & 10.68 & 7.21 & 89.58 & 7.21 & 8.11 & 15.06 & 18.66 \\
\hline
\end{tabular}

$\mathrm{TSH}=$ thyroid stimulating hormone; FT4 = free thyroxine.

\section{Positive cutoff value and reference ranges of TPOAb in 7-12 weeks gestation}

In this study, there were a total of 584 cases of pregnant women who had a normal reference range of TSH levels that were between 0.01 and $3.67 \mathrm{mU} / \mathrm{L}$. After calculating this for pregnant women, the 90th percentile value and the positive cutoff value of TPOAb were determined. Hence, we can make a conclusion that the TPOAb positive cutoff value for 611 cases of pregnant women at 7 to 12 weeks gestation is $206.77 \mathrm{mU} / \mathrm{L}$, and that the reference range is $0-206.77 \mathrm{mU} / \mathrm{L}$.

In this study, 66 cases of the total participants have TPOAb $>206.77 \mathrm{mU} / \mathrm{L}$, and the TPOAb positive rate was $10.8 \%(66 / 611)$. If we calculate the TPOAb-positive rate according to the reference standard (TPOAb $>60 \mathrm{mU} / \mathrm{L}$ ), which is provided by manufacturer, the TPOAb-positive rate of 611 women who are in early pregnancy is $22.9 \%(140 / 611)$. This result is a 1 -fold increase from the previous estimation.

\section{Effect of TPOAb on TSH and FT4 levels in women at 7 to 12 weeks gestation}

Regression analysis showed that TPOAb and TSH were positively correlated, and that TPOAb and FT4 had a negative correlation. The values of $\beta$ are 1.406 and 0.820 , respectively. The $95 \%$ CI values are $(1.349,1.413)$ and $(0.805,0.835)$, respectively. P values for both were 0.000 .

The TSH median value of 66 cases of TPOAb-positive pregnant women was 1.51 $\mathrm{mU} / \mathrm{L}$ and the TSH median value of $545 \mathrm{TPOAb}$-negative pregnant women was $1.11 \mathrm{mU} / \mathrm{L}$. The difference was statistically significant $(\mathrm{P}=0.000)$. Based on this data, we know that when comparing the median value, the former is $0.4 \mathrm{mU} / \mathrm{L}$ higher than the latter. For 8 cases $(12.12 \%)$ of TPOAb-positive pregnant women whose TSH $>3.67 \mathrm{mU} / \mathrm{L}$, and 15 cases $(2.75 \%)$ of TPOAb-negative pregnant women whose TSH $>3.67 \mathrm{mU} / \mathrm{L}$, the former TSH levels abnormally elevated risk by 4.4 -fold more than the latter (Figure 1).

For 66 cases of pregnant women who were TPOAb-positive, the FT4 median value was $10.55 \mathrm{pM}$. For 545 cases of TPOAb-negative pregnant women, the FT4 median value was $10.68 \mathrm{pM}$. The differences were not significantly different $(\mathrm{P}=0.319)$ (Figure 2). 


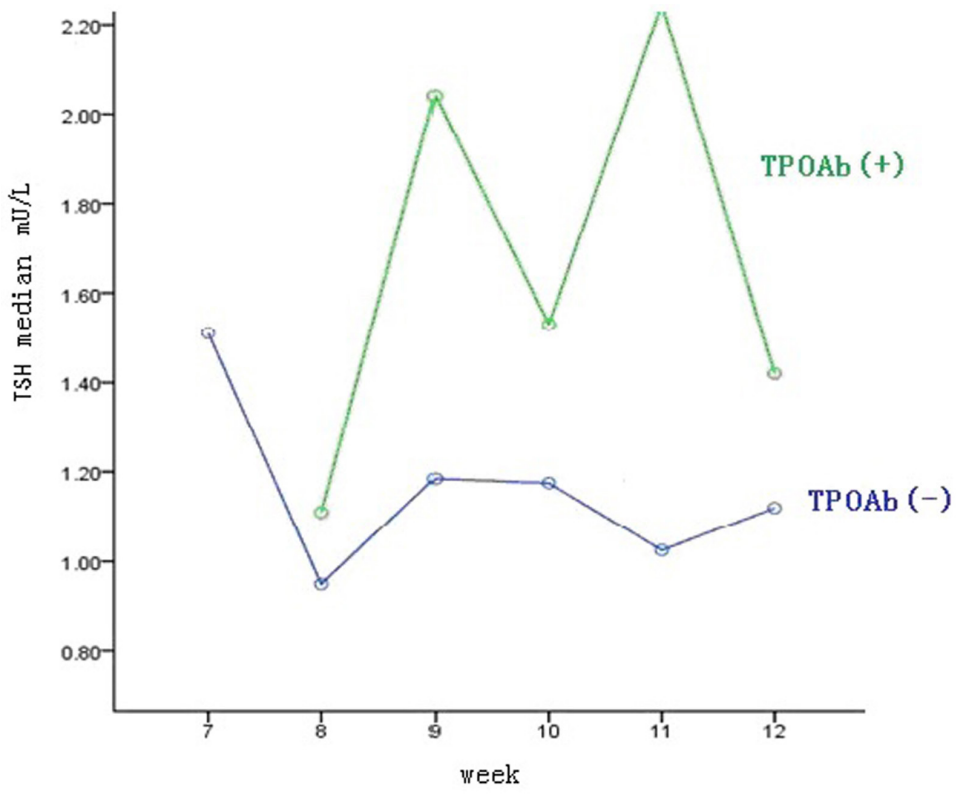

Figure 1. Median value of TPOAb positive is significant higher than TPOAb negative in early pregnancy but different gestational.

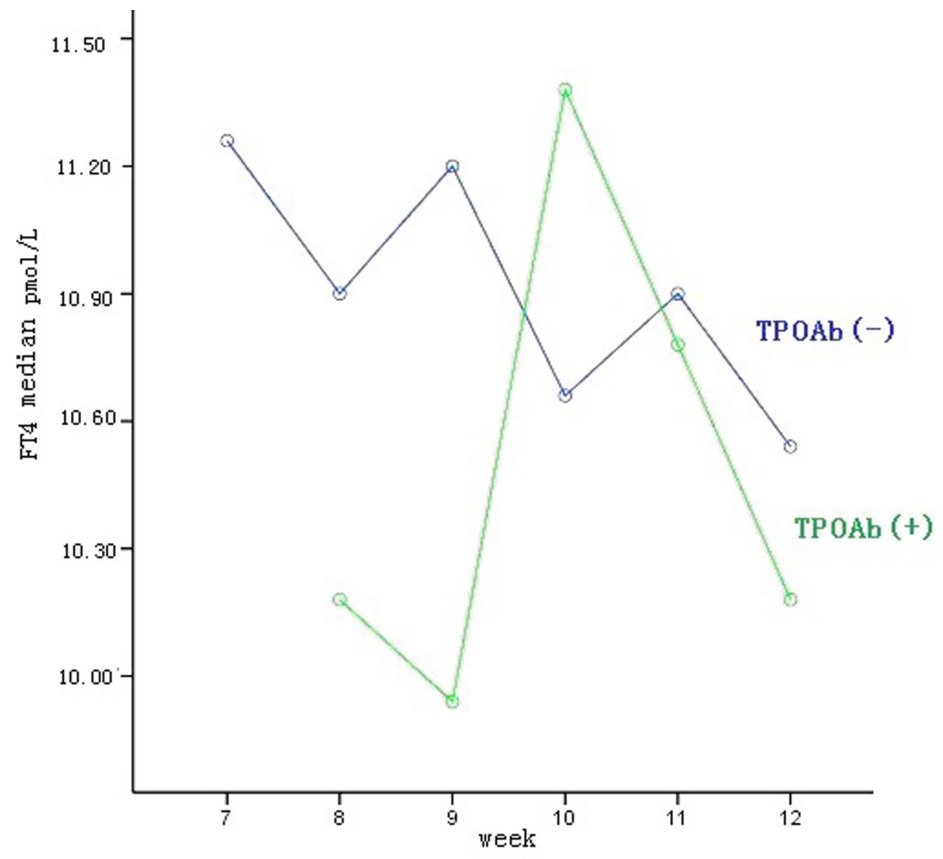

Figure 2. FT4 median value of TPOAb positive and TPOAb negative has no significant difference in early pregnancy but different gestational. 


\section{DISCUSSION}

\section{Effect of TPOAb on gestational thyroid function}

Thyroid peroxidase (TPO) is a key enzyme in thyroid hormone synthesis, as well as an autoantigen that can induce AITD. In the immune response, the combination of TPO and $\mathrm{TPOAb}$ can cause progressive thyroid cell damage, leading to hypothyroidism lesions that are often hidden. TPOAb is considered to be a marker of early thyroid dysfunction. TPOAb is positive in approximately 10 to $15 \%$ of the normal population, among which about $2 \%$ have subclinical hypothyroidism that progresses to clinical hypothyroidism. Pregnant women with TPOAb-positivity are prone to inadequate secretion of thyroid hormone, due to the decline in thyroid compensatory ability. In women with TPOAb positivity before pregnancy, the risk of hypothyroidism and subclinical hypothyroidism after pregnancy will increase by 2 -fold.

Springer et al. (2009) showed that for pregnant women with normal thyroid function in the first trimester, those with TPOAb-positivity have higher levels of TSH, and $44.1 \%$ of those with TSH values above the upper limits have TPOAb-positivity. In this study, the TSH median values among TPOAb-positive women was elevated by $0.4 \mathrm{mU} / \mathrm{L}$ more than TPOAbnegative ones, and the risks of abnormally elevated TSH is increased by 4.4-fold, which is similar to the $0.43 \mathrm{mU} / \mathrm{L}$ elevated TSH median reported by Negro et al. (2011).

As a strong risk factor for miscarriage, premature delivery, placental abruption, perinatal death, and gestational diabetes, TPOAb is associated with adverse pregnancy outcomes. The latest guidelines of the American Thyroid Association (ATA) in 2011 suggested that TPOAb-positive pregnant women should be tested for TSH levels from 4 to 6 weeks, using the reference range of gestational age-specific thyroid function to assess thyroid function.

\section{Setting a standard gestational TPOAb reference range}

The U.S. National Academy of Clinical Biochemistry (NACB) guidelines (Baloch et al., 2003) recommend that when we want to develop the TPOAb reference intervals, the "standard crowd" should be 120 "normal subjects" free from any history or family history of thyroid disease, having serum TSH levels 0.5 and $2.0 \mathrm{mIU} / \mathrm{L}$, male, $<30$ years of age. Because of special changes in thyroid function during pregnancy, non-pregnancy TPOAb reference standards do not apply to pregnancy; therefore, there is no unity of the TPOAb reference range of non-pregnancy.

Due to different background populations (race, age, fertility), laboratory tests, and statistical methods, the TPOAb-positive rate for pregnant women will be significantly different in different studies. Gilbert et al. (2008) reported an early pregnancy TPOAb-positive rate of $15.7 \%$, with the TPOAb reference standard provided by the manufacturer. Männistö et al. (2011) reported a TPOAb-positive rate of 5.0\%, and the TPOAb reference standard was obtained from specific reference intervals of pregnant population. Based on the results, we know that the TPOAb-positive rate will often be higher when it is calculated according to manufacturer standards. Springer et al. (2009) set the TPOAb standard as the 90th percentile of the normal reference range population in early pregnancy. When testing 5281 cases of women in early pregnancy, the TPOAb-positive rate is about $11.2 \%$ according to the standard mentioned above. The TPOAb-positive rate is $22.1 \%$ based on the standard provided by manufacturers. 
Therefore, the latter is higher than the former at one time. In this study, the positive rate of TPOAb is $22.1 \%$ at 8 to 12 gestational weeks according to the manufacturer standard (TPOAb $>60 \mathrm{mIU} / \mathrm{L}$ ). Based on the Springer statistical method, we corrected the TPOAb positive cutoff value to $206.77 \mathrm{mIU} / \mathrm{L}$ and, after that, the positive rate of TPOAb was calculated as $10.8 \%$. This result is consistent with the TPOAb positive rate in Springer' study when the cut-off of anti-TPO was established at $143 \mathrm{kU} / 1$. Whether or not this statistical method for TPOAb cutoff value is more reasonable for the clinic, it needs long follow-up study to explore the effect of $\mathrm{TPOAb}$ on the pregnancy outcome in mothers and children.

The storage methods used for laboratory samples also affect TPOAb test results. Männistö et al. (2007) reported in a previous study that the TPOAb levels seem higher when serum samples were preserved for a longer period of time. However, the levels of TSH and FT4 do not change significantly. We recommend that the thyroid function standard of population-specific reference should be developed during pregnancy to avoid overestimation of the TPOAbpositive rate.

In addition, elevated $\beta$-HCG will suppress TSH levels in the early stages of pregnancy. Some scholars have suggested that the development of a TSH normal reference range in early pregnancy should be excluded if the $\beta-\mathrm{HCG}$ is greater than 3 times the mean value (Springer et al., 2009). Although we did not detect $\beta$-HCG in the study population during early pregnancy, pregnant women with elevated $\beta$-HCG levels cannot be ruled out. To some extent, this might affect the accuracy of the TSH reference interval.

\section{Should routine screening for TPOAb in early pregnancy or L-T4 intervention in AITD patients with normal thyroid function be used?}

Some scholars have suggested that early pregnancy screening for thyroid dysfunction by the indicators of TSH, FT4, and TPOAb can reduce the misdiagnosis rate of mild hypothyroidism in pregnancy (Burman, 2009; Shan et al., 2009). The 2007 NACB Guide (de Groot et al., 2012) pointed out that in the first three months of pregnancy and before pregnancy, screening for thyroid dysfunction using serum TSH and TPOAb detection to find mild hypothyroidism (TSH $>4.0 \mathrm{mIU} / \mathrm{L}$ ) and to assess the risk of thyroiditis is very important. The results of this study show that TPOAb often presents in early pregnant women with no previous history of thyroid disease, and that the positive rate can reach up to about $10 \%$. The risk of abnormal elevation of TSH in TPOAb-positive pregnant women is 4.4-fold greater than in TPOAb-negative pregnant women. This demographic of AITD patients will be missed without early pregnancy screening for TPOAb. Hence, our study supports routine screening for $\mathrm{TPOAb}$ during early pregnancy.

There are still some controversial issues in academia, such as whether there is a need for the intervention of L-thyroxine (L-T4) in pregnant women who have normal thyroid function but are TPOAb-positive, how to use L-T4, and whether it affects the offspring. In 2010, Reid et al. conducted a meta-analysis of three prospective randomized controlled trials and the results showed: 1) L-T4 intervention therapy does not reduce the incidence of preeclampsia, but can reduce the incidence of preterm birth, 2) L-T4 intervention treatment did not show a trend of reducing spontaneous abortion, and 3) the three studies did not include a long-term assessment of the impact on the intelligence of children. In 2011, the ATA pointed out in the latest clinical guidelines (Männistö et al., 2011) that there is a lack of sufficient evidence 
to support or refute routine screening of TPOAb in early pregnancy, and it remains unclear whether there is a need for L-T4 intervention in AITD patients with normal thyroid function. As a result, we look forward to a large, prospective, randomized controlled study to provide sufficient evidence.

\section{ACKNOWLEDGMENTS}

Research supported by the Starting Scientific Program of Beijing Friendship Hospital affiliated to Capital Medical University (\#YYQDKT2010-11), the Beijing Municipal Science and Technology Commission Research Projects (\#D101100050010033), and the Capital Health Development Research (\#2011-1002-04).

\section{REFERENCES}

Baloch Z, Carayon P, Conte-Devolx B, Demers LM, et al. (2003). Laboratory medicine practice guidelines. Laboratory support for the diagnosis and monitoring of thyroid disease. Thyroid 13: 3-126.

Burman KD (2009). Controversies surrounding pregnancy, maternal thyroid status, and fetal outcome. Thyroid 19: 323 326.

de Groot L, Abalovich M, Alexander EK, Amino N, et al. (2012). Management of thyroid dysfunction during pregnancy and postpartum: an Endocrine Society clinical practice guideline. J. Clin. Endocrinol. Metab. 97: 2543-2565.

Gilbert RM, Hadlow NC, Walsh JP, Fletcher SJ, et al. (2008). Assessment of thyroid function during pregnancy: firsttrimester (weeks 9-13) reference intervals derived from Western Australian women. Med. J. Aust. 189: 250-253.

Haddow JE, Cleary-Goldman J, McClain MR, Palomaki GE, et al. (2010). Thyroperoxidase and thyroglobulin antibodies in early pregnancy and preterm delivery. Obstet. Gynecol. 116: 58-62.

Lepoutre T, Debiève F, Gruson D and Daumerie C (2012). Reduction of miscarriages through universal screening and treatment of thyroid autoimmune diseases. Gynecol. Obstet. Invest. 74: 265-273.

Männistö T, Surcel HM, Bloigu A, Ruokonen A, et al. (2007). The effect of freezing, thawing, and short- and long-term storage on serum thyrotropin, thyroid hormones, and thyroid autoantibodies: implications for analyzing samples stored in serum banks. Clin. Chem. 53: 1986-1987.

Männistö T, Surcel HM, Ruokonen A, Vaarasmaki M, et al. (2011). Early pregnancy reference intervals of thyroid hormone concentrations in a thyroid antibody-negative pregnant population. Thyroid 21: 291-298.

Negro R and Stagnaro-Green A (2011). Thyroid autoantibodies, preterm birth, and miscarriage. BMJ 342: d2260.

Reid SM, Middleton P, Cossich MC and Crowther CA (2010). Interventions for clinical and subclinical hypothyroidism in pregnancy. Cochrane Database Syst. Rev. CD007752.

Shan ZY, Chen YY, Teng WP, Yu XH, et al. (2009). A study for maternal thyroid hormone deficiency during the first half of pregnancy in China. Eur. J. Clin. Invest. 39: 37-42.

Springer D, Zima T and Limanova Z (2009). Reference intervals in evaluation of maternal thyroid function during the first trimester of pregnancy. Eur. J. Endocrinol. 160: 791-797. 